\title{
ARTICLE
}

\section{Ultrafast optical modification of exchange interactions in iron oxides}

\author{
R.V. Mikhaylovskiy ${ }^{1,2}$, E. Hendry ${ }^{1}$, A. Secchi ${ }^{2}$, J.H. Mentink ${ }^{3}$, M. Eckstein ${ }^{3}$, A. Wu ${ }^{4}$, R.V. Pisarev ${ }^{5}$, V.V. Kruglyak ${ }^{1}$, \\ M.I. Katsnelson', Th. Rasing ${ }^{2} \&$ A.V. Kimel ${ }^{2}$
}

Ultrafast non-thermal manipulation of magnetization by light relies on either indirect coupling of the electric field component of the light with spins via spin-orbit interaction or direct coupling between the magnetic field component and spins. Here we propose a scenario for coupling between the electric field of light and spins via optical modification of the exchange interaction, one of the strongest quantum effects with strength of $10^{3}$ Tesla. We demonstrate that this isotropic opto-magnetic effect, which can be called inverse magneto-refraction, is allowed in a material of any symmetry. Its existence is corroborated by the experimental observation of terahertz emission by spin resonances optically excited in a broad class of iron oxides with a canted spin configuration. From its strength we estimate that a sub-picosecond modification of the exchange interaction by laser pulses with fluence of about $1 \mathrm{~mJ} \mathrm{~cm}-2$ acts as a pulsed effective magnetic field of 0.01 Tesla.

\footnotetext{
${ }^{1}$ School of Physics, University of Exeter, Stocker Road, Exeter EX4 4QL, UK. ${ }^{2}$ Radboud University Nijmegen, Institute for Molecules and Materials, Heyendaalseweg 135, 6525 AJ, Nijmegen, The Netherlands. ${ }^{3}$ University of Hamburg, Center for Free-Electron Laser Science, Luruper Chaussee 149,22761 Hamburg, Germany. ${ }^{4}$ Shanghai Institute of Ceramics, Chinese Academy of Sciences, Shanghai 200050, China. ${ }^{5}$ loffe Physical-Technical Institute, Russian Academy of Sciences, 194021 St Petersburg, Russia. Correspondence and requests for materials should be addressed to R.V.M.

(email: R.Mikhaylovskiy@science.ru.nl).
} 


\section{T} he symmetric part of the exchange interaction between spins is responsible for the very existence of magnetic ordering ${ }^{1}$. It is described by the Hamiltonian $\hat{\mathrm{H}}_{\mathrm{ex}}=J \sum_{i, j}\left(\hat{\mathbf{S}}_{i} \cdot \hat{\mathbf{S}}_{j}\right)$, where $J$ is the exchange integral; $\hat{\mathrm{S}}_{i}$ and $\hat{\mathrm{S}}_{j}$ are the spins of the $i$ th and $j$ th adjacent magnetic ions. The antisymmetric part $\hat{\mathrm{H}}_{\mathrm{DM}}=2 \mathbf{D} \cdot \sum_{i, j}\left(\hat{\mathbf{S}}_{i} \times \hat{\mathbf{S}}_{j}\right)$, characterized by a vector parameter $\mathbf{D}$ and called Dzyaloshinskii-Moriya interaction, gives rise to canted antiferromagnetism ${ }^{2-3}$ in iron oxides.

The ability to control the exchange interaction by light has intrigued researchers in many areas of physics, ranging from quantum computing ${ }^{4-6}$ to strongly correlated materials ${ }^{7-9}$. Laser-induced heating ${ }^{10-11}$ and photo-doping 9,12 have been suggested to cause a modification of the exchange interaction. However, these phenomena rely on the absorption of light and are neither universal, that is, they are only present in specific materials, nor direct, that is, not instantaneous. Recently the time-resolved evolution of the exchange splitting in magnetic metals $\mathrm{Ni}$ and $\mathrm{Gd}$ subjected to ultrafast laser excitation was measured using photoelectron spectroscopy ${ }^{13}$ and angleresolved photoemission ${ }^{14}$ techniques, respectively. Both of these techniques, unfortunately, do not allow to distinguish the intrinsic dynamics of the exchange parameters such as $J$ from the demagnetization dynamics. Nevertheless, a direct, truly ultrafast effect of the electric field of light on the exchange interaction must be feasible in any material. In a medium of arbitrary symmetry, such an effect may be expressed phenomenologically by introducing an isotropic term in the Hamiltonian of the two-photon interaction between the light and spins

$$
\hat{\mathrm{H}}_{\mathrm{IMR}}=I_{\mathrm{opt}} \alpha \sum_{i, j}\left(\hat{\mathbf{S}}_{i} \cdot \hat{\mathbf{S}}_{j}\right)+2 I_{\mathrm{opt}} \boldsymbol{\beta} \cdot \sum_{i, j}\left(\hat{\mathbf{S}}_{i} \times \hat{\mathbf{S}}_{j}\right),
$$

where $I_{\mathrm{opt}}$ is the intensity of light; $\alpha$ and $\boldsymbol{\beta}$ are some scalar and vector coefficients, respectively. The presence of the interaction Hamiltonian (1) manifests itself as a magnetic refraction, described by an isotropic contribution to the dielectric permittivity $\varepsilon_{\mathrm{IMR}} \sim M^{2}$ that leads to a dependence of the refractive index on the magnitude of the magnetization $M^{15,16}$. The first term in the Hamiltonian describes the intensity dependent contribution, $\Delta J=\alpha I_{\text {opt }}$, to the symmetric Heisenberg exchange integral $J$, whereas the second term describes the intensity dependent contribution, $\Delta \mathbf{D}=\boldsymbol{\beta} I_{\text {opt }}$, to the Dzyaloshinskii-Moriya vector $\mathbf{D}$. Recently the effect of isotropic magneto-refraction has been used to probe d-f exchange in EuTe ${ }^{17}$. As for other magneto-optical phenomena, isotropic magnetorefraction must be connected with an inverse effect ${ }^{18}$ described by the same Hamiltonian (1), that is, the optical generation of a torque $\mathbf{T}_{i}$ acting on a spin $\mathbf{S}_{i}$ due to the light-induced perturbation of the exchange parameters

$$
\mathbf{T}_{i}=-\gamma\left(\mathbf{S}_{i} \times \frac{\partial \hat{\mathrm{H}}_{\mathrm{IMR}}}{\partial \mathbf{S}_{i}}\right)=-\gamma \Delta J\left(\mathbf{S}_{i} \times \mathbf{S}_{j}\right)-2 \gamma\left(\mathbf{S}_{i} \times\left(\mathbf{S}_{j} \times \Delta \mathbf{D}\right)\right),
$$

where $\gamma$ is the absolute value of the gyromagnetic ratio. The torque (2) is zero in materials with collinear magnetic configurations since $\left(\mathbf{S}_{i} \times \mathbf{S}_{j}\right)=0$. In contrast to the torques exerted by the optical perturbation of the spin-orbit interaction ${ }^{19-21}$ or transient magnetic field $^{22-23}$, it is independent of the light polarization.

In a broad class of transition metal oxides the magnetic order is governed by indirect exchange via ligand ions (superexchange) ${ }^{1}$ and is defined by virtual charge-transfer transitions of electrons between ligands and magnetic ions. Hence, one can anticipate the feasibility of a direct effect of the electric field of light on the exchange energy via virtual or real excitation of specific optical transitions that modify the hopping of the electrons between electronic orbitals centred at the transition metal ions and oxygen ligands, respectively.
Antiferromagnetic iron oxides possessing weak ferromagnetism, such as iron borate $\mathrm{FeBO}_{3}$, rare-earth orthoferrites $\mathrm{RFeO}_{3}$ ( $\mathrm{R}$ stands for a rare-earth element) and hematite $\alpha-\mathrm{Fe}_{2} \mathrm{O}_{3}$, are natural candidates for observing such ultrafast optical modification of the superexchange interactions. In these compounds the $\mathrm{Fe}^{3+}$ ions (spin quantum number $S=5 / 2$ and orbital momentum quantum number $L=0$ ) form two magnetic sublattices, the spins of which are antiferromagnetically coupled $^{24}$. The presence of the Dzyaloshinskii-Moriya antisymmetric exchange interaction leads to a slight canting of the spins from the antiparallel orientation by an angle of $\sim 0.5-1^{\circ}$. The value of the canting is defined by the ratio $D / J$ between the antisymmetric and symmetric exchange parameters. Thus, one could expect that an ultrafast optical perturbation of the exchange parameters could also change the ratio $D / J$ and thereby trigger, by the torque defined in equation (2), the socalled quasi-antiferromagnetic resonance mode. This mode corresponds to oscillations of the magnitude of the weak magnetic moment without a change of its orientation ${ }^{25}$. According to equations (1) and (2), the ultrafast optical perturbation of the exchange parameters in these weak ferromagnets is an isotropic mechanism, that is, it can excite the quasi-antiferromagnetic resonance independently from the light polarization and propagation direction. The excited oscillating magnetic dipole in turn will lead to the generation of terahertz $(\mathrm{THz})$ radiation which can be measured using terahertz emission spectroscopy ${ }^{26}$, as has been demonstrated before in experiments with ferromagnetic metals ${ }^{27-29}$ and antiferromagnetic insulators $\mathrm{NiO}^{30-33}$ and $\mathrm{MnO}^{34}$. In the present context, observation of $\mathrm{THz}$ emission due to laser excitation of the quasi-antiferromagnetic spin resonance via an isotropic mechanism would indicate an ultrafast manipulation of the exchange interactions. Importantly, to observe the $\mathrm{THz}$ radiation the emitting dipole must lie in the plane of the sample and therefore be perpendicular to the propagation direction of light ${ }^{26}$.

Here we reveal the inverse magneto-refractive effect to be responsible for ultrafast modulation of the superexchange interaction in a very broad class of canted antiferromagnets. Our findings are supported by a low-energy theory for the magnetic interactions between non-equilibrium electrons subjected to an external time-dependent electric field. We present quantitative estimates of the strength and timescale of the optical perturbation of the exchange parameters.

\section{Results}

THz emission from weak ferromagnets. Recently we reported measurements of $\mathrm{THz}$ emission signals in the rare-earth orthoferrites ${ }^{26} \mathrm{TmFeO}_{3}$ and $\mathrm{ErFeO}_{3}$ which revealed the optical excitation of the high-frequency quasi-antiferromagnetic mode in these compounds along with the low-frequency quasiferromagnetic mode, another form of the antiferromagnetic resonance in canted antiferromagnets which involves the precession of the magnetization with no change in its length ${ }^{25}$. We also observed unexpected weak modes at $\sim 0.3 \mathrm{THz}$ and assigned them to paramagnetic impurities ${ }^{26}$. The measurements suggested that the quasi-antiferromagnetic mode must be excited via a polarization-independent mechanism of coupling between light and spins. However, the data were not sufficient to identify the exact nature of the opto-magnetic excitation, in general, and to relate it to an optical perturbation of the exchange parameters $D / J$ via inverse magneto-refractive effect, in particular.

To demonstrate the existence of the inverse magnetorefractive effect described above, and in particular the polarization-independent ultrafast optical perturbation of the exchange parameters $\mathrm{D} / \mathrm{J}$, we have studied the $\mathrm{THz}$ emission from a single $\mathrm{FeBO}_{3}$ cut perpendicularly to the $z$-crystallographic axis 
so that it lacks significant in-plane anisotropy of both optical and magnetic properties. The magnetization lying in the plane of the sample was aligned horizontally by a constant bias magnetic field of $\sim 0.1 \mathrm{~T}$. The sample was illuminated by $\sim 100$-fs laser pulses with their photon energy centred at $1.55 \mathrm{eV}$. We performed time-resolved detection of the $\mathrm{THz}$ radiation emitted from the sample in the direction of the $z$ axis (see Fig. 1a). The waveforms generated at different temperatures are shown in Fig. $1 b$. We observe that the optical excitation of the sample leads to quasi-monochromatic emission at a frequency of $\sim 0.45 \mathrm{THz}$ (Fig. 1c), which corresponds to the frequency of the quasiantiferromagnetic mode in $\mathrm{FeBO}_{3}$ (ref. 35). The amplitude of the oscillations gradually decreases as the temperature approaches the Néel temperature $T_{N} \sim 350 \mathrm{~K}$ (see Supplementary Fig. 1).

To confirm that a similar mechanism is also present in other weak ferromagnets, we performed more detailed measurements of $\mathrm{THz}$ emission from orthoferrites similar to those reported in ref. 26, but for a temperature range in which only the quasiantiferromagnetic mode was excited, making the interpretation of the experimental data less complex. Fig. 1e,d demonstrate that below $55 \mathrm{~K}$ the $\mathrm{TmFeO}_{3}$ single crystal plate cut perpendicularly to the $z$-crystallographic axis emits radiation with only one spectral component at the frequency of $\sim 0.8 \mathrm{THz}$, which is the frequency of the quasi-antiferromagnetic mode ${ }^{25}$ in $\mathrm{TmFeO}_{3}$ (see also Supplementary Fig. 1). To check that the observed effect is not due to the specific electronic structure of $\mathrm{Tm}^{3+}$ ions, we have performed similar experiments on the $\mathrm{YFeO}_{3}$ single crystal cut perpendicularly to the $x$-crystallographic axis (see Fig. 2). Fig. 2 shows that using an ultrafast optical excitation we are able to excite oscillations at a frequency of $\sim 0.55 \mathrm{THz}$, which again corresponds to the frequency of the quasi-antiferromagnetic mode in $\mathrm{YFeO}_{3}$ (ref. 25) (see also Supplementary Fig. 2). We have also observed similar polarization-insensitive ultrafast optical excitation of the quasi-antiferromagnetic mode in $y$-and $x$-cut samples of $\mathrm{ErFeO}_{3}$ (see Fig. 3), $x$-cut and $y$-cut $\mathrm{DyFeO}_{3}$ and in hematite $\alpha-\mathrm{Fe}_{2} \mathrm{O}_{3}$ (see Supplementary Figs 3 and 4; Supplementary Note 1).

Properties of the $\mathrm{THz}$ emission. To determine if the excitation mechanism is isotropic, we performed a set of measurements to systematically investigate its dependence on fluence and polarization of the laser pulse and found that the oscillation amplitudes depend linearly on the intensity of the pump (see Supplementary Figs 1 and 2) and are insensitive to the pump polarization (see Supplementary Fig. 5). By comparing the signals generated in the crystals pumped along different crystallographic directions, such as $y$ and $x$ axes in $\mathrm{ErFeO}_{3}$ (shown in Fig. 3) one can see that the excitation mechanism is isotropic with respect to the pump propagation direction as well. The phase of the measured oscillations changed by $\pi$ with the reversal of the magnetization direction, confirming the magnetic origin of the signals (see Fig. 4). Moreover, this shows that the direction of the light-

a
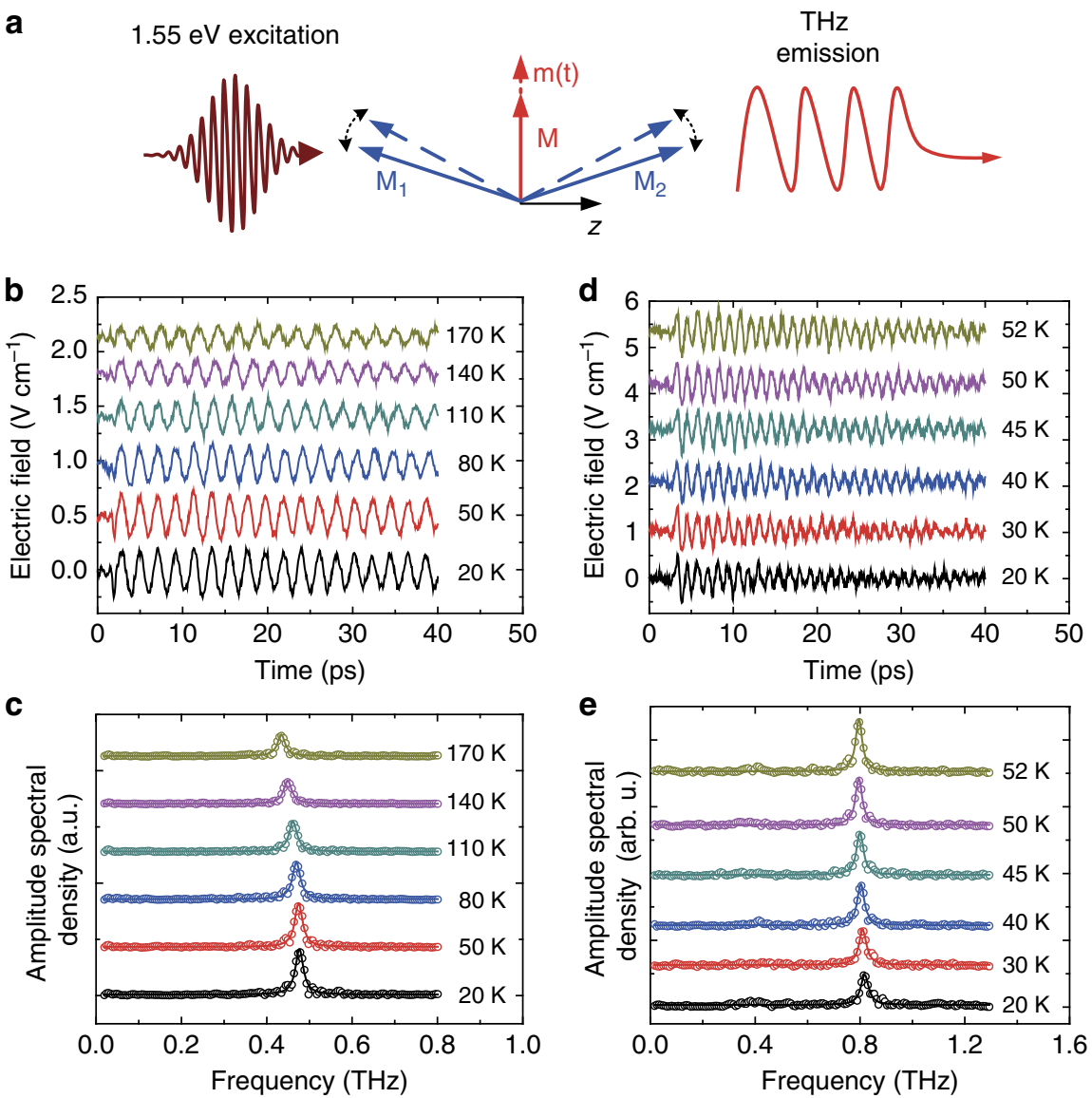

Figure 1 | Terahertz emission generated in $\mathbf{F e B O}_{3}$ and $\mathbf{T m F e O}_{3}$. (a) The magnetization $\mathbf{M}=\mathbf{M}_{1}+\mathbf{M}_{2}$ lies in the plane of the crystal sample plate. The optical pump is focused onto the sample plate along its normal ( $z$ axis), while the THz emission is collected along the same direction at the opposite side of the sample. The $\mathrm{THz}$ emission arises from the quasi-antiferromagnetic oscillations $\mathbf{m}(t)$. (b) $\mathrm{The} \mathrm{FeBO}_{3}$ emission at different temperatures below $170 \mathrm{~K}$. The zero time delay corresponds to an arbitrary starting position. The laser pulse arrives just before the commencement of the oscillations. (c) The spectra of the $\mathrm{FeBO}_{3}$ emission obtained from the data by Fourier transform (open circles) fitted by Lorentzian functions (solid lines). (d) The $\mathrm{TmFeO}_{3}$ emission at different temperatures below $55 \mathrm{~K}$. (e) The $\mathrm{TmFeO}_{3}$ emission spectra (open circles) fitted with Lorentzian functions (solid lines). 
induced torque exciting the quasi-antiferromagnetic oscillations is determined by the orientation of the spins, and not by the polarization of light. All these observations are in perfect qualitative agreement with the anticipated features of an isotropic mechanism of optical modification of the exchange interaction described by equation (1).
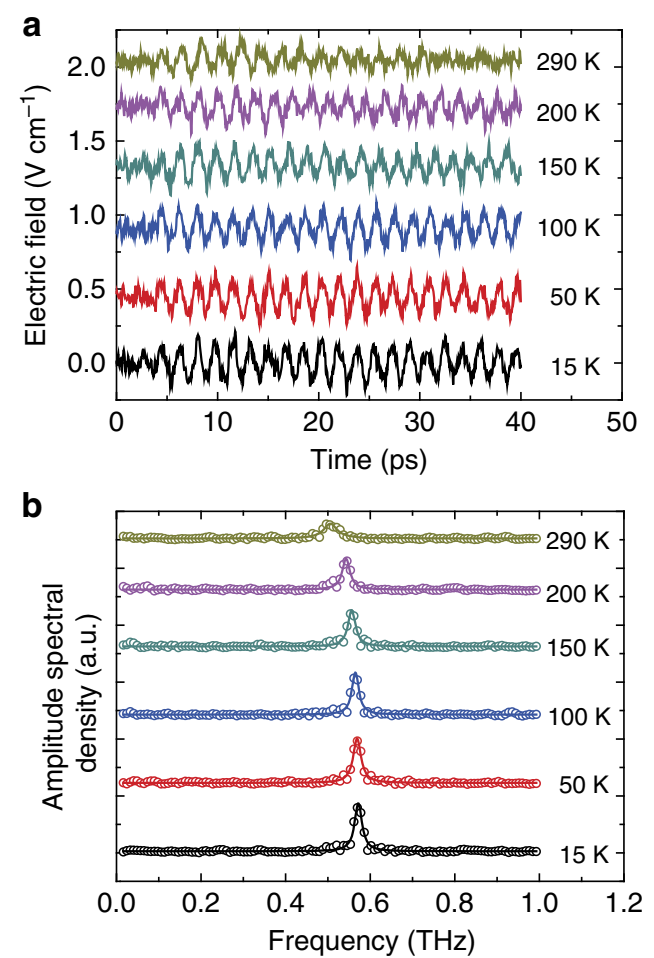

Figure 2 | Terahertz emission generated in $\mathbf{Y F e O}_{3}$. (a) The emission waveforms generated in the $x$ cut $\mathrm{YFeO}_{3}$ sample at different temperatures. (b) The spectra of the $\mathrm{YFeO}_{3}$ emission obtained from the data by Fourier transform (open circles) fitted by Lorentzian functions (solid lines).

\section{Discussion}

The consistent observation of the photo-excitation of the quasi-antiferromagnetic mode in a range of compounds clearly indicates that this effect originates from the perturbation of the $D / J$ ratio. The isotropic and polarization-insensitive character of the excitation rules out mechanisms based on the inverse Faraday effect $^{19}$, which is sensitive to the ellipticity of the pump, or the inverse Cotton-Mouton effect ${ }^{36}$, which is sensitive to the polarization direction of the pump relative to the magnetization direction. We note that the $\mathrm{THz}$ emission observed from the antiferromagnets $\mathrm{NiO}$ (refs 30-33) and $\mathrm{MnO}$ (ref. 34) did not contain a contribution that was isotropic relative to the pump polarization. Indeed the Dzyaloshinskii-Moriya antisymmetric exchange interaction is not allowed in these cubic insulators $\mathrm{NiO}$ and $\mathrm{MnO}$ and in the absence of an external magnetic field the torque (2) is equal to zero. Moreover, the observed effect cannot be attributed to the laser-induced change of the magnetocrystalline anisotropy as demonstrated in garnets ${ }^{37}$ and orthoferrites $^{38-39}$ since this mechanism can trigger only the low-frequency quasi-ferromagnetic mode. This conclusion is further corroborated by the observation of this effect in $\mathrm{FeBO}_{3}$, which lacks significant in-plane anisotropy.

We would like to note that our demonstration of an ultrafast change of the ratio between the exchange parameters is based on the observation of the femtosecond excitation of the quasi-antiferromagnetic mode of spin resonance. Despite several optical pump-probe spectroscopy experiments on femtosecond laser excitation of spins in the orthoferrites and iron borate, the optical excitation of the quasi-antiferromagnetic mode has been very rarely observed. The very first observation of ultrafast laser excitation of both quasi-ferromagnetic and quasi-antiferromagnetic modes was reported for $\mathrm{DyFeO}_{3}$ in ref. 19 and later confirmed by Satoh et al. ${ }^{40}$ It was found, however, that for the chosen crystallographic orientation of the crystals the mechanisms of the excitation were dominated by the polarization dependent inverse Faraday and inverse Cotton-Mouton effects. Due to the fact that $\mathrm{DyFeO}_{3}$ was a strongly anisotropic material, discerning the helicity independent contribution from the data were not possible. Later studies only revealed the possibility of femtosecond helicity dependent excitation of the quasi- a

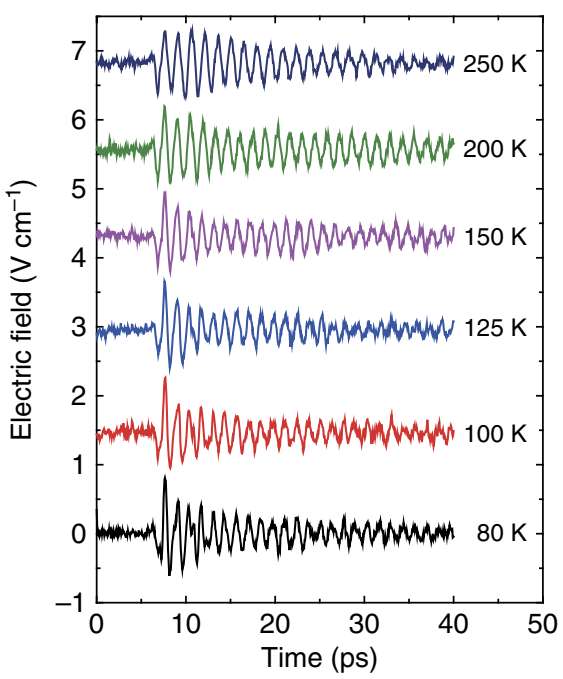

b

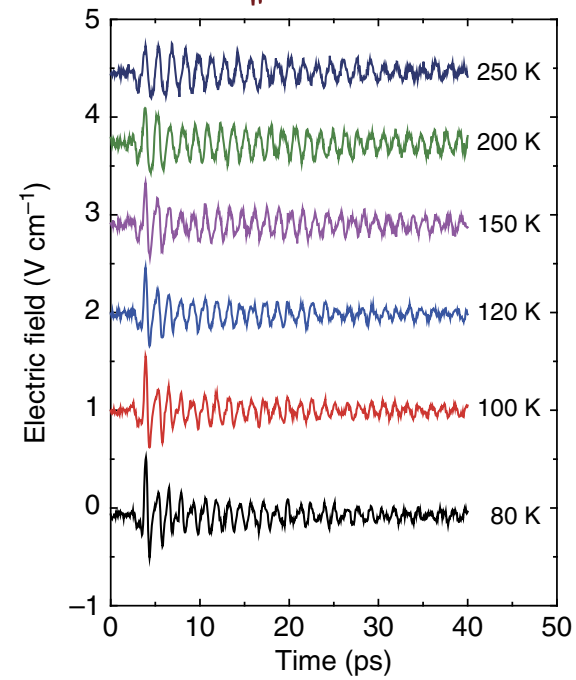

Figure 3 | Terahertz emission generated in $\mathbf{E r F e O}_{3}$. (a) The $\mathrm{ErFeO}_{3}$ emission at different temperatures below room temperature generated by the optical pulses propagating along the $y$ axis. (b) The $\mathrm{ErFeO}_{3}$ emission generated by the optical pulses propagating along the $x$ axis. 
ferromagnetic mode in $\mathrm{TmFeO}_{3}$ (ref. 39), $\mathrm{HoFeO}_{3}$ (ref. 41), $\mathrm{FeBO}_{3}$ (ref. 36), $\mathrm{ErFeO}_{3}$ (ref. 42), and $\mathrm{SmPrFeO}_{3}$ (ref. 43). As a result of laser-induced heating and a subsequent spinreorientation phase transition, an ultrafast excitation of again the quasi-ferromagnetic mode was reported for $\mathrm{TmFeO}_{3}$ (ref. 38), $\mathrm{ErFeO}_{3}$ (ref. 42) and $\mathrm{SmFeO}_{3}$ (ref. 44). No optically induced spin dynamics was reported for hematite.

The main reason why the isotropic, polarization-independent effect, reported here has not been observed before is that the detection in the aforementioned experiments was based on the magneto-optical Faraday effect which probes the spins indirectly that is, it strongly relies on the magneto-optical susceptibility and does not provide a direct picture of spin dynamics. Using $\mathrm{THz}$ emission spectroscopy, which is a more direct probe of the oscillating magnetization ${ }^{26}$, we have been able to identify the isotropic contribution to the optical excitation of the quasiantiferromagnetic spin resonance, which is the principal result of this paper. We also point out that the excitation of the quasiantiferromagnetic mode via the inverse Faraday effect is possible only in samples with the magnetization pointing out-of-plane. However in this geometry the $\mathrm{THz}$ waves cannot be emitted from the sample, hence we do not observe inverse Faraday-like effects in our $\mathrm{THz}$ signals.

To specify the possible optical transitions responsible for our observations, we note that the dispersion of the refraction coefficient for all these compounds is dominated by the offresonant susceptibilities related to the electric dipole allowed charge-transfer transitions between the $2 p$ orbitals of oxygen and the $3 d$ orbitals of the $\mathrm{Fe}^{3+}$ ions above $3 \mathrm{eV}$ (refs 45-47). During the laser pulse duration and the time of optical decoherence, the collective electron wave-functions are coherent superpositions of the wave-functions of the ground and excited states. Such
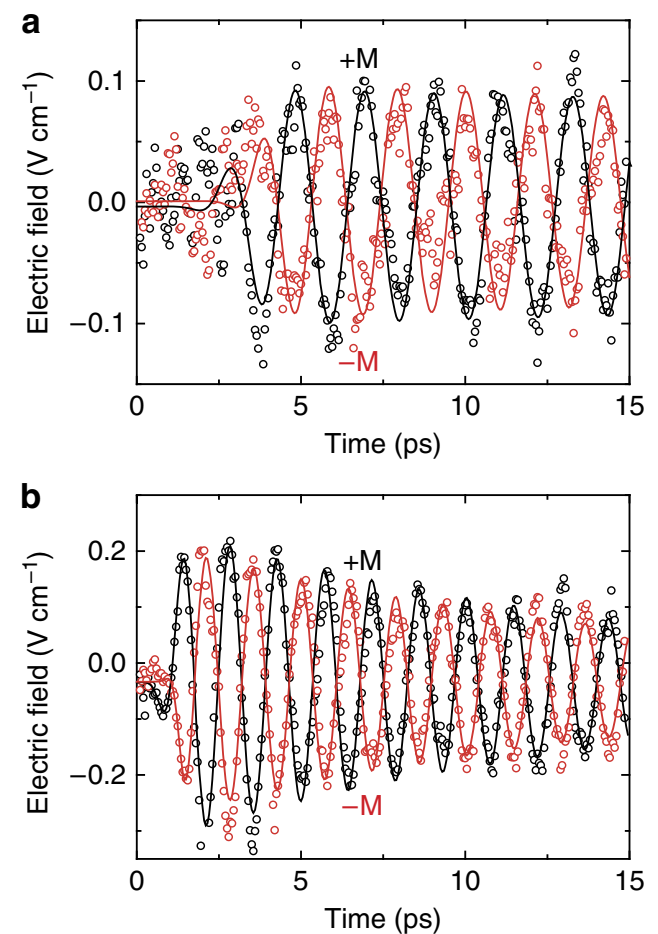

Figure 4 | $\mathrm{THz}$ emission versus the magnetization direction in $\mathrm{FeBO}_{3}$ and $\mathbf{E r F e O}_{3}$. (a) The $\mathrm{THz}$ electric field emitted from the $\mathrm{FeBO}_{3}$ sample for opposite orientations of the magnetization (black and red open circles). (b) The $\mathrm{THz}$ electric field emitted from the $x$ cut $\mathrm{ErFeO}_{3}$ sample for opposite orientations of the magnetization. The data are fitted with exponentially decaying sinusoids multiplied by error functions (solid lines). ultrafast modification of the wave-functions affects the exchange interaction between the spins of the neighbouring $\mathrm{Fe}^{3+}$ ions and thus changes the energy of the superexchange interaction (see Fig. 5). One can therefore expect that the observed effect of light on the exchange interaction is inherent to all magnetic materials, the magnetic order of which is governed by superexchange. However, only when the spins are canted, either by the Dzyaloshinskii-Moriya interaction or by an applied magnetic field, such an ultrafast change of the exchange interaction will lead to excitation of the antiferromagnetic resonance and the subsequent emission of $\mathrm{THz}$ radiation in accord with equation (2).

Our data are in excellent agreement with the phenomenology of equation (1) that gives the simplest and most plausible explanation. A possible microscopic scenario underpinning the phenomenology of our results can be understood in the framework of a recently developed formalism ${ }^{48}$ for microscopic magnetic interactions out of equilibrium (see Methods sections and Supplementary Note 2). To demonstrate the effect of a femtosecond laser pulse on the super-exchange interaction we numerically evaluated the time-dependent exchange for a 3-ion $\mathrm{Fe}^{3+}-\mathrm{O}^{2-}-\mathrm{Fe}^{3+}$ cluster, which is characterized by a strong on-site Coulomb interaction $U$ on the $\mathrm{Fe}^{3+}$ ions, an energy level shift $\Delta$ between the $\mathrm{Fe}^{3+}$ and $\mathrm{O}^{2-}$ ions, and an equilibrium
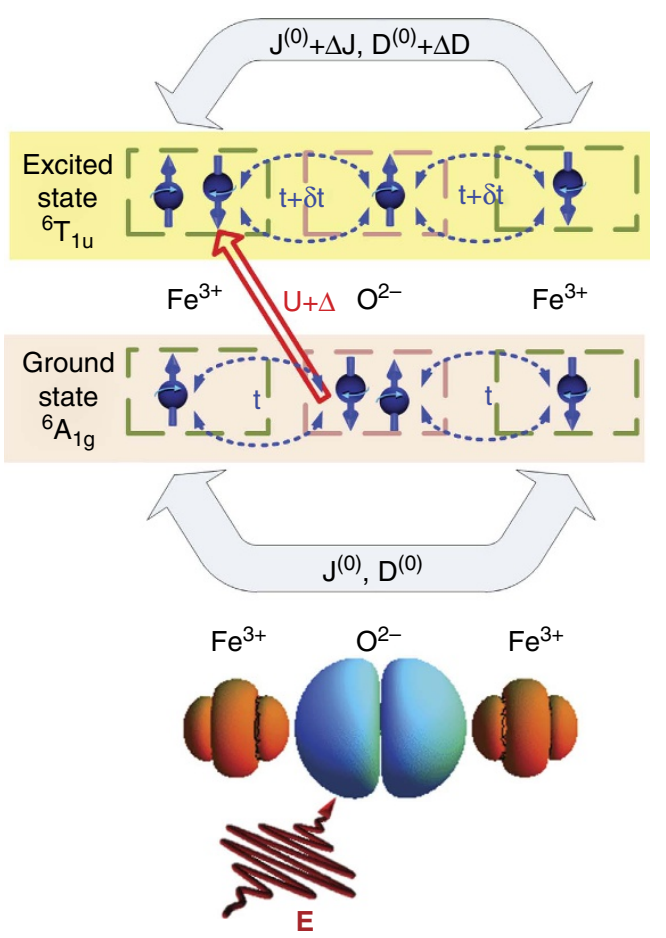

Figure 5 | The mechanism for optical modulation of the exchange in iron oxides. In the ground state ${ }^{6} \mathrm{~A}_{1 \mathrm{~g}}$ the exchange interactions $f^{(0)}$ and $D^{(0)}$ between the iron $\mathrm{Fe}^{3+}$ spins are mediated by the spins of the oxygen $\mathrm{O}^{2-}$ ions and occur due to the virtual hopping $t$ (shown with dashed blue arrows) of electrons (blue balls with arrows schematically showing the 'up' and 'down' directions of their spins) within the iron-oxygen cluster. The strong electric field $\mathbf{E}$ of the laser pulse of arbitrary polarization excites virtual electric-dipole transitions from the ground state ${ }^{6} \mathrm{~A}_{1 \mathrm{~g}}$ to the excited state ${ }^{6} \mathrm{~T}_{1 \mathrm{u}}$ over the energy gap $U+\Delta$ in the iron-oxygen cluster. The transitions involve the charge transfer (red arrow) from the oxygen to the iron site thereby changing the hopping amplitude $t+\delta t$ and the electronic wave functions. As a result the exchange parameters are modified to $J^{(0)}+\Delta J$ and $D^{(0)}+\Delta D$. 
hopping amplitude $t_{0}$ between $\mathrm{Fe}$ and $\mathrm{O}$ ions. For a small ratio $t_{0} / U$, the leading-order expression for the equilibrium superexchange in this system $\operatorname{reads}^{49} J=\frac{2 t_{0}^{4}}{U_{1}^{2}}\left(\frac{1}{U}+\frac{1}{U_{1}}\right)$, where $U_{1}=U+\Delta$. By gradually switching on an oscillating offresonant electric field we observe an enhancement of the exchange interaction proportional to the intensity of the laser pulse (see Supplementary Figs 6 and 7; Supplementary Note 2). To further understand the dependence of the superexchange on the laser field, we studied analytically a periodically driven cluster model. The shift of the energy levels under the periodic driving field can be understood within Floquet theory ${ }^{50}$ (see Supplementary Note 2), which gives an analytical expression for the change of the exchange interaction:

$\Delta J=\frac{\varepsilon^{2} t_{0}^{4}}{2}\left(\sum \pm\left[\frac{1}{U_{1} \pm \hbar \omega}+\frac{1}{U_{1}}\right]^{2} \frac{1}{U \pm \hbar \omega}-\frac{4}{U_{1}^{2} U}-\frac{4}{U_{1}^{3}}\right)$.

Here, $\varepsilon=e a E_{0} / \hbar \omega$ is the amplitude of the vector potential that describes the electric field in the Coulomb gauge with amplitude $E_{0}, e$ and $a$ are the unit charge and lattice constant, respectively, and $\omega$ is the frequency of the optical field. The terms dependent on $\pm \omega$ are the photon-assisted charge transfer excitations, while the last two terms describe a laser-induced decrease of the effective hopping amplitude within the $\mathrm{Fe}^{3+}-\mathrm{O}^{2-}-\mathrm{Fe}^{3+}$ cluster by a coherent destruction of tunnelling ${ }^{51}$. We obtain excellent quantitative agreement of $\Delta J / J$ between equation (3) and the numerical results obtained from the general theory (see Supplementary Note 2). In the experiment we typically have $\hbar \omega \sim U_{1} / 2$, from which we conclude that the strengthening of the exchange interaction is caused by a photon-assisted chargetransfer excitation, as illustrated in Fig. 5. Using typical experimental parameters $U=3 \mathrm{eV}, \Delta=0.25 \mathrm{eV}, t_{0}=0.5 \mathrm{eV}$ and $\hbar \omega=1.5 \mathrm{eV}$, we find that an optical pulse with a fluence of $1 \mathrm{~mJ} \mathrm{~cm}{ }^{-2}$ and a corresponding electric field amplitude $E_{0}=0.12 \mathrm{~V} \AA^{-1}$ should induce an increase of the exchange integral $\Delta J / J$ of over $1 \%$. Our model analysis neither incorporates multi-orbital effects nor a description of the non-equilibrium Dzyaloshinskii-Moriya interaction, which certainly would be beyond the scope of this report. Importantly, we have shown theoretically that the optical manipulation of magnetic interactions is feasible already in the elementary super-exchange model defined by the $\mathrm{Fe}-\mathrm{O}-\mathrm{Fe}$ cluster.

To determine whether laser excitation leads to a decrease or an increase of the ratio $D / J$ we take advantage of the strong temperature dependence of the magnetic anisotropy, which is characteristic for many orthoferrites. For instance, heating of $\mathrm{TmFeO}_{3}$ from 80 to $90 \mathrm{~K}$ leads to a change of the equilibrium orientation of the weak magnetic moment from the $x$ to the $z$ axis. If the equilibrium orientation is changed as a result of a sudden heating by a femtosecond laser pulse, such a change is followed by oscillations of the weak magnetic moment in the $(x z)$ plane at the frequency of the quasi-ferromagnetic mode $(\sim 100 \mathrm{GHz})^{38-39}$. As discussed in ref. 26 in the range between 55 and $68 \mathrm{~K}$, such low-frequency oscillations corresponding to the quasi-ferromagnetic mode are observed in $\mathrm{THz}$ emission spectra together with the high-frequency quasi-antiferromagnetic oscillations (see Fig. 6). We applied a low pass filter to the data (cutoff frequency $250 \mathrm{GHz}$ ) to isolate the quasi-ferromagnetic mode and a high-frequency filter (cutoff frequency $650 \mathrm{GHz}$ ) to isolate the quasi-antiferromagnetic mode. Such a choice of the cutoffs ensures the filtering out of the impurity modes which complicate the dynamics ${ }^{26}$. It is seen from Fig. 6 that the high-frequency mode measured at $60 \mathrm{~K}$ is in phase with that observed at $40 \mathrm{~K}$. One can also see that the initial phases of the

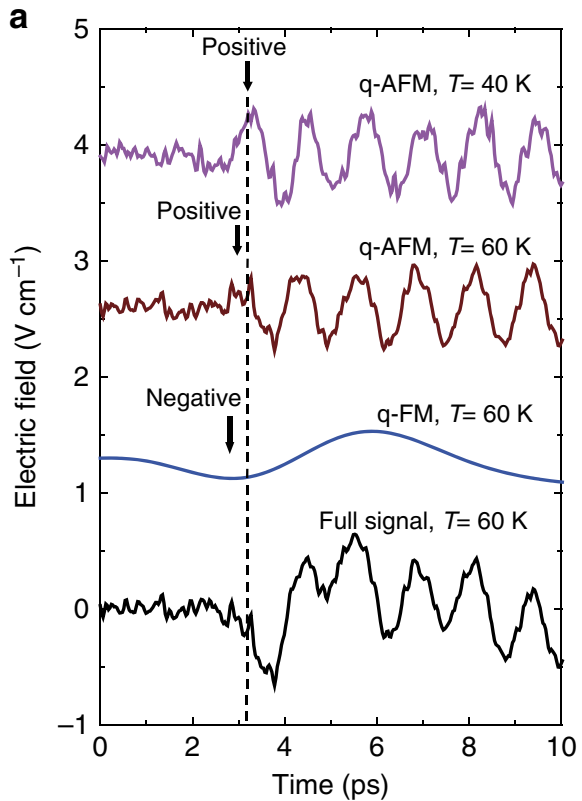

b
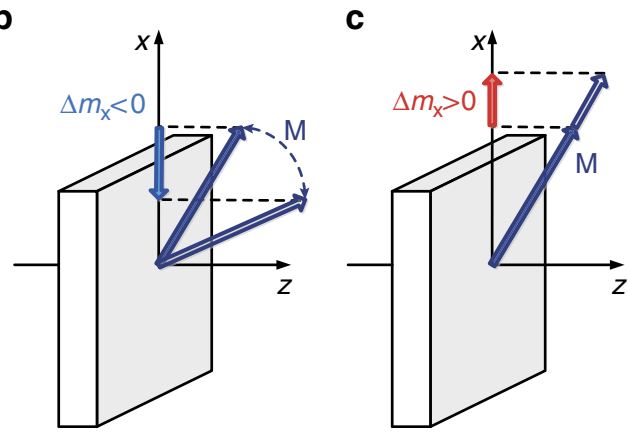

Figure 6 | Determination of the absolute sign of the change of $D / J$ in $\mathrm{TmFeO}_{3}$. (a) The signal emitted just below the spin reorientation temperature at $60 \mathrm{~K}$ (black) is shown together with its low-frequency (blue) and high-frequency part (brown). The latter part is in phase with the signal measured at $40 \mathrm{~K}$ which describes a quasi-antiferromagnetic (q-AFM) mode only (magenta). The low-frequency part corresponds to the quasi-ferromagnetic mode ( $q-F M)$. The first half-cycle of the quasiantiferromagnetic mode has a different sign compared with the first halfcycle of the quasi-ferromagnetic mode (see dashed line). (b) During the spin reorientation the spin configuration of $\mathrm{TmFeO}_{3}$ continuously rotates in the $(x z)$ plane, while keeping the weak ferromagnetic moment in the same plane. At low temperatures, the magnetization is oriented along the $x$ axis. So, due to the laser-induced reorientation at $60 \mathrm{~K}$, the $x$-component of the magnetization initially decreases. (c) Since the first half-cycle of the quasiantiferromagnetic mode has a different sign, due to the exchange-driven torque the magnetization initially moves so as to have a positive $x$-component which implies an increase of $D / J$.

low-frequency quasi-ferromagnetic and high-frequency quasiantiferrimagnetic modes are $\sim 180^{\circ}$ apart. Note that for the $z$-cut $\mathrm{TmFeO}_{3}$ sample, with a net magnetic moment oriented upwards, a laser-induced spin-reorientation transition should trigger the quasi-ferromagnetic mode in such a way that the $M_{x}$ component of the magnetization decreases. The observed difference in the phases between the two oscillations shows that the quasiantiferromagnetic mode is triggered in such a way that the $M_{x}$ component increases, which means that the canting angle becomes larger. Such a behaviour can only be explained by assuming that the quasi-antiferromagnetic oscillations are 
triggered by an increase of the ratio of the exchange parameters $D / J$. If this conclusion is true, in the $x$-cut sample the initial phases of the two modes must be the same, since the spin reorientation in this sample proceeds in the opposite direction. Measurements in the vicinity of the spin-reorientation temperature in $\mathrm{ErFeO}_{3}$ cut perpendicular to the $x$ axis confirm this conclusion (see Supplementary Fig. 8; Supplementary Note 3 ). Interestingly, the increase of the ratio $D / J$ cannot be explained on the basis of the simplistic model defined by the $\mathrm{Fe}-\mathrm{O}-\mathrm{Fe}$ cluster that predicts an increase of $J$ and does not evaluate the change of $D$. However, the calculation of $\Delta J$ demonstrates the plausibility of the proposed mechanism of optical manipulation of the symmetric exchange interaction in principle.

To deduce the magnitude and timescale of the exchange modification from the experimental data, we have solved the Maxwell equations for a slab of a material with an oscillating magnetization triggered by a perturbation of the ratio $D / J$ and calculated the electromagnetic radiation emitted by the slab into the free space. A quantitative analysis supports the subpicosecond impact on the spin system (see Supplementary Fig. 9; Supplementary Notes 4 and 5). The absence of any significant broadband $\mathrm{THz}$ emission, which must accompany a laser-induced ultrafast demagnetization ${ }^{27-28}$ in iron borate and the orthoferrites (Fig. 1), supports the claim that femtosecond changes of the net magnetic moment can be neglected. The fact that the observed spin dynamics do not arise from the laser-induced heating is evidenced by the absence of a correlation between the strength of the observed signals and the specific heat and thermal conductivity of the studied materials. For example, the specific heat of $\mathrm{YFeO}_{3}$ below $100 \mathrm{~K}$ grows rapidly as the temperature increases while its thermal conductivity exhibits a pronounced peak around $30 \mathrm{~K}$ (ref. 52). At the same time the efficiency of the quasi-antiferromagnetic mode excitation in this compound does not depend on temperature at all (see Supplementary Fig. 2a). The observation of the very same effect of comparable strength in hematite with high optical absorption $\sim 2,000 \mathrm{~cm}^{-1}$ at $1.55 \mathrm{eV}$ (ref. 53), in the orthoferrites with moderate optical absorption $\sim 200 \mathrm{~cm}^{-1}$ at $1.55 \mathrm{eV}$ (ref. 45) and in virtually transparent iron borate with absorption $<100 \mathrm{~cm}^{-1}$ at $1.55 \mathrm{eV}$ (ref. 54) shows that the optical modification of the $D / J$ does not rely on laser heating due to optical absorption.

The maximum value of the oscillating magnetization in the samples is estimated to be $\sim 1 \mathrm{Am}^{-1}$. Oscillations with such an amplitude can only be triggered if the laser excitation results in an ultrafast increase of the ratio $D / J$ by $>0.01 \%$ (see Supplementary Notes 4 and 5). Taking into account the parameters of our experiment, one can find that the sub-picosecond laser excitation with a fluence of $\sim 1 \mathrm{~mJ} \mathrm{~cm}{ }^{-2}$ changes the potential energy of the magnetic system by $\sim 1 \mu \mathrm{J} \mathrm{cm}^{-2}$ and acts as an effective magnetic field pulse of $\sim 0.01 \mathrm{~T}$ (see Supplementary Note 6). These values (normalized to the optical fluence) correspond to some of the largest effects of light on magnetic systems observed to date $\mathrm{e}^{19,22}$.

To summarize, the demonstrated feasibility of a subpicosecond modification of the fundamental exchange parameters $J$ and $D$ and the ratio between them opens wide prospects for optical control of magnetically ordered materials. The suggested mechanism is not restricted by any requirement on the crystal symmetry and must thus be applicable to other classes of magnetic materials. Given that in some materials isotropic magneto-refraction can be significantly larger than that in iron oxides, we foresee many opportunities to enhance the effects reported here. Finally, we anticipate that by tuning the wavelength of light, one should be able to affect selectively different exchange parameters in magnetic materials.

\section{Methods}

Samples. The crystals used in the present study were grown by floating zone melting (orthoferrites) and from the gas phase (iron borate and hematite) The orthoferrite samples were $60-100-\mu \mathrm{m}$ thick and cut perpendicularly to the $z$ axis $\left(\mathrm{TmFeO}_{3}\right)$, the $x$ axis $\left(\mathrm{YFeO}_{3}, \mathrm{ErFeO}_{3}\right)$ and the $y$ axis, $\left(\mathrm{ErFeO}_{3}, \mathrm{DyFeO}_{3}\right)$. The iron borate $\mathrm{FeBO}_{3}$ sample $\left(370-\mu \mathrm{m}\right.$ thick) and haematite $\alpha-\mathrm{Fe}_{2} \mathrm{O}_{3}$ sample $(500-\mu \mathrm{m}$ thick) were cut perpendicularly to the $z$ axis. The lateral size of all plates was $\sim 5 \mathrm{~mm}$.

Terahertz spectrometer. A conventional time-domain $\mathrm{THz}$ spectrometer was used in the measurements. The THz spectrometer was powered by a Ti:sapphire amplified laser, emitting a sequence of optical pulses $(800 \mathrm{~nm}$ wavelength, $100 \mathrm{fs}$ duration) with the repetition frequency of $1 \mathrm{kHz}$. Each laser pulse was divided into a stronger pump pulse and a weaker probe pulse. The pump spot size was larger than the aperture in the sample holder $(\sim 2 \mathrm{~mm}$ in diameter $)$ to provide a quasiuniform excitation with a fluence of $\sim 1 \mathrm{~mJ} \mathrm{~cm}^{-2}$. The electric field of the emitted $\mathrm{THz}$ wave was measured by the electro-optical sampling technique. The sample was held inside a closed cycle, helium cryostat $\left(15-300 \mathrm{~K}, 10^{-4} \mathrm{mbar}\right)$.

Theory of non-equilibrium exchange interactions. We use a general formalism in which magnetic interactions are obtained from a purely electronic model by introducing small time-dependent rotations of the spin quantization axes as was recently described in (ref. 48). For the general non-equilibrium case the evolution of the electronic model is described using the Schwinger-Keldysh/Kadanoff-Baym non-equilibrium action and partition function, with the effective action written in terms of Grassmann fields. By integrating over the electronic degrees of freedom in the rotated reference frame, an effective quadratic spin model is obtained in which the time-dependent exchange interaction parameters are identified from the mapping of the effective action to a time-dependent classical Heisenberg model. The resulting expressions turn out to be combinations of non-equilibrium electronic Green's functions and self-energies, which have to be evaluated numerically to assess the modification of exchange interaction by time-dependent perturbations of the electron model.

This method is implemented for the simplest model system that exhibits the physics of superexchange, which consists of a chain of three atoms, labelled as 0,1 and 2 . Atoms 0 and 2 correspond to transition metal sites with one partially filled $d$ orbital and atom 1 contributes one filled (oxygen) $p$ orbital. The Hamiltonian consists of a local part $H_{\mathrm{loc}}$ and a time-dependent hopping term $H^{\prime}(t)$ :

$$
\begin{gathered}
H_{\mathrm{loc}}=\varepsilon_{d} \sum_{\sigma}\left(n_{0 \sigma}+n_{2 \sigma}\right)+\varepsilon_{p} \sum_{\sigma} n_{1 \sigma}+U \sum_{j=0,2} n_{j \uparrow} n_{j \downarrow}, \\
H^{\prime}(t)=-\sum_{j=0,1} \sum_{\sigma} t_{0} \mathrm{e}^{\mathrm{i} \varphi(t)} c_{j \sigma}^{+} c_{j+1 \sigma}+h . c .
\end{gathered}
$$

Here $c_{j \sigma}^{+}$creates an electron with spin $\sigma=\{\uparrow, \downarrow\}$ at site $j$, and $n_{j \sigma}=c_{j \sigma}^{+} c_{j \sigma}$ is the number operator. The parameters $\varepsilon_{d}$ and $\varepsilon_{p}$ are the orbital energies of $d$ and $p$ orbitals, respectively, and $U$ is the local (Hubbard) interaction energy associated with $d$ orbitals. $H^{\prime}(t)$ accounts for hopping between $p$ and $d$ orbitals; $t_{0}$ is the equilibrium hopping parameter, while $\varphi(t)$ is the time-dependent Peierls phase, which absorbs the effect of the time-dependent electric field. In the Coulomb gauge and for a spatially uniform vector potential the Peierls phase is given by

$$
\varphi(t)=\frac{e a}{\hbar c} A_{\|}(t)
$$

where $A_{\|}(t)$ is the component of the vector potential parallel to the chain and $a$ is the lattice spacing. In the chosen gauge, the electric field is related to the vector potential as $\mathbf{E}(t)=-\frac{1 \partial}{\partial \partial t} \mathbf{A}(t)$. In the experimentally relevant regime the model is characterized by $U, U+\varepsilon_{d}-\varepsilon_{p} \gg t_{0}$ and a total filling of four electrons. To compute the non-equilibrium functions, this cluster model is solved numerically using exact diagonalization of the time-dependent Schrödinger equation. The electric field is taken as the product of an oscillatory component and a gradually changing envelope function with a rising time in the order of $\sim 10$ periods of oscillation. The frequency $\omega$ of the oscillating part is below the charge-transfer gap, which prevents direct charge-transfer transitions, consistently with the experimental conditions.

Further details related to the theoretical and numerical methods are discussed in Supplementary Note 2 .

\section{References}

1. Stöhr, J. \& Siegmann, H. C. Magnetism: From Fundamentals to Nanoscale Dynamics (Springer, 2006).

2. Dzyaloshinskii, I. E. A thermodynamic theory of "weak" ferromagnetism of antiferromagnetics. J. Phys. Chem. Solids 4, 241-255 (1958).

3. Moriya, T. Anisotropic superexchange interaction and weak ferromagnetism. Phys. Rev 120, 91-98 (1960).

4. Duan, L.-M., Demler, E. \& Lukin, M. D. Controlling spin exchange interactions of ultracold atoms in optical lattices. Phys. Rev. Lett. 91, 090402 (2003).

5. Trotzky, S. et al. Time-resolved observation and control of superexchange interactions with ultracold atoms in optical lattices. Science 319, 295-299 (2008). 
6. Chen, Y.-A. et al. Controlling correlated tunneling and superexchange interactions with ac-driven optical lattices. Phys. Rev. Lett. 107, 210405 (2011).

7. Wall, S., Prabhakaran, D., Boothroyd, A. T. \& Cavalleri, A. Ultrafast coupling between light, coherent lattice vibrations, and the magnetic structure of semicovalent $\mathrm{LaMnO}_{3}$. Phys. Rev. Lett. 103, 097402 (2009).

8. Först, M. et al. Driving magnetic order in a manganite by ultrafast lattice excitation. Phys. Rev. B 84, 241104 (2011).

9. Li, T. et al. Femtosecond switching of magnetism via strongly correlated spin-charge quantum excitations. Nature 496, 69-73 (2013).

10. Ju, G. et al. Ultrafast generation of ferromagnetic order via a laser-induced phase transformation in FeRh thin films. Phys. Rev. Lett. 93, 197403 (2004).

11. Thiele, J. U., Buess, M. \& Back, C. H. Spin dynamics of the antiferromagneticto-ferromagnetic phase transition in FeRh on a sub-picosecond time scale. Appl. Phys. Lett. 85, 2857-2959 (2004).

12. Mentink, J. H. \& Eckstein, M. Ultrafast quenching of the exchange interaction in a Mott insulator. Phys. Rev. Lett. 113, 057201 (2014).

13. Rhie, H.-S., Dürr, H. A. \& Eberhardt, W. Femtosecond electron and spin dynamics in Ni/W(110) films. Phys. Rev. Lett. 90, 247201 (2003).

14. Carley, R. et al. Femtosecond laser excitation drives ferromagnetic gadolinium out of magnetic equilibrium. Phys. Rev. Lett. 109, 057401 (2012).

15. Markovin, P. A., Pisarev, R. V., Smolensky, G. A. \& Syrnikov, P. P. Observation of isotropic magnetic contribution to the refractive index of $\mathrm{ABF}_{3}$-type cubic crystals. Solid State Commun. 19, 185-188 (1976).

16. Demokritov, S. O., Kreines, N. M. \& Kudinov, V. I. Observation of a new light-scattering mechanism in a antiferromagnet. JETP Lett. 41, 46-49 (1985).

17. Subkhangulov, R. R. et al. All-optical manipulation and probing of the $\mathrm{d}-\mathrm{f}$ exchange interaction in EuTe. Sci. Rep. 4, 4368 (2014).

18. Kirilyuk, A., Kimel, A. V. \& Rasing, T. h. Ultrafast optical manipulation of magnetic order. Rev. Mod. Phys. 82, 2731-2784 (2010).

19. Kimel, A. V. et al. Ultrafast non-thermal control of magnetization by instantaneous photomagnetic pulses. Nature 435, 655-657 (2005).

20. Satoh, T. et al. Directional control of spin-wave emission by spatially shaped light. Nat. Photonics 6, 662-666 (2012).

21. Tesařová, N. et al. Experimental observation of the optical spin-orbit torque. Nat. Photonics 7, 492-498 (2013).

22. Kampfrath, T. et al. Coherent terahertz control of antiferromagnetic spin waves. Nat. Photonics 5, 31-34 (2011)

23. Vicario, C. et al. Off-resonant magnetization dynamics phase-locked to an intense phase-stable terahertz transient. Nat. Photonics 7, 720-723 (2013).

24. White, R. L. Review of recent work on the magnetic and spectroscopic properties of the rare-earth orthoferrites. J. Appl. Phys. 40, 1061-1069 (1969).

25. Srinivasan, G. \& Slavin, A. N. (Ed.) High frequency processes in magnetic materials (World Scientific Publishing, 1995).

26. Mikhaylovskiy, R. V. et al. Terahertz emission spectroscopy of laser-induced spin dynamics inTmFeO $\mathrm{F}_{3}$ and $\mathrm{ErFeO}_{3}$ orthoferrites. Phys. Rev. B 90, 184405 (2014).

27. Beaurepaire, E. et al. Coherent terahertz emission from ferromagnetic films excited by femtosecond laser pulses. Appl. Phys. Lett. 84, 3465-3467 (2004).

28. Hilton, D. J. et al. Terahertz emission via ultrashort-pulse excitation of magnetic metal films. Opt. Lett. 29, 1805-1807 (2004).

29. Kampfrath, T. et al. Terahertz spin current pulses controlled by magnetic heterostructures. Nat. Nanotechnol. 8, 256-260 (2013).

30. Nishitani, J., Kozuki, K., Nagashima, T. \& Hangyo, M. Terahertz radiation from coherent antiferromagnetic magnons excited by femtosecond laser pulses. Appl. Phys. Lett. 96, 221906 (2010).

31. Higuchi, T., Kanda, N., Tamaru, H. \& Kuwata-Gonokami, M. Selection rules for light-induced magnetization in a crystal with threefold symmetry: the case of antiferromagnetic NiO. Phys. Rev. Lett. 106, 047401 (2011).

32. Kanda, N. et al. The vectorial control of magnetization by light. Nat. Commun. 2, 362 (2011).

33. Nishitani, J., Nagashima, T. \& Hangyo, M. Coherent control of terahertz radiation from antiferromagnetic magnons in $\mathrm{NiO}$ excited by optical laser pulses. Phys. Rev. B 85, 174439 (2012).

34. Nishitani, J., Nagashima, T. \& Hangyo, M. Terahertz radiation from antiferromagnetic $\mathrm{MnO}$ excited by optical laser pulses. Appl. Phys. Lett. 103, 081907 (2013).

35. Polupanov, V. N., Dakhov, N. F., Kiselyev, V. K. \& Seleznev, V. N. Investigation of iron borate on the submillimeter waves. Int. J. Infrared Millimet. Waves 16, 1167-1172 (1995).

36. Kalashnikova, A. M. et al. Impulsive generation of coherent magnons by linearly polarized light in the easy-plane antiferromagnet $\mathrm{FeBO}_{3}$. Phys. Rev. Lett. 99, 167205 (2007).

37. Hansteen, F., Kimel, A. V., Kirilyuk, A. \& Rasing, T. h. Femtosecond photomagnetic switching of spins in ferrimagnetic garnet films. Phys. Rev. Lett. 95, 047402 (2005)

38. Kimel, A. V., Kirilyuk, A., Tsvetkov, A., Pisarev, R. V. \& Rasing, T. h. Laser-induced ultrafast spin reorientation in the antiferromagnet $\mathrm{TmFeO}_{3}$. Nature 429, 850-853 (2004).
39. Kimel, A. V. et al. Optical excitation of antiferromagnetic resonance in $\mathrm{TmFeO}_{3}$. Phys. Rev. B 74, 060403R (2006).

40. Iida, R. et al. Spectral dependence of photoinduced spin precession in $\mathrm{DyFeO}_{3}$ Phys. Rev. B 84, 064402 (2011).

41. Kimel, A. V. et al. Inertia-driven spin switching in antiferromagnets. Nat. Phys 5, 727-731 (2009).

42. Jong, J. A., de, Kimel, A. V., Pisarev, R. V., Kirilyuk, A. \& Rasing, T. h. Laser-induced ultrafast spin dynamics in $\mathrm{ErFeO}_{3}$. Phys. Rev. B 84, 10442 (2011).

43. de Jong, J. A. et al. Coherent control of the route of an ultrafast magnetic phase transition via low-amplitude spin precession. Phys. Rev. Lett. 108, 157601 (2012).

44. Guyader, L. L. e. et al. Dynamics of laser-induced spin reorientation in $\mathrm{Co} / \mathrm{SmFeO}_{3}$ heterostructure. Phys. Rev. B 87, 054437 (2013).

45. Wood, D. L., Remeika, J. P. \& Kolb, E. D. Optical spectra of rare-earth orthoferrites. J. Appl. Phys. 41, 5315-5322 (1970).

46. Lichtenstein, A. I., Moskvin, A. S. \& Gubanov, V. A. Electronic structure of $\mathrm{Fe}^{3+}$-centers and exchange interactions in rare-earth orthoferrites. Solid State Phys. 24, 3596 (1982).

47. Pisarev, R. V., Moskvin, A. S., Kalashnikova, A. M. \& Rasing, T. h. Charge transfer transitions in multiferroic $\mathrm{BiFeO}_{3}$ and related ferrite insulators. Phys. Rev. B 79, 235128 (2009).

48. Secchi, A., Brener, S., Lichtenstein, A. I. \& Katsnelson, M. I. Non-equilibrium magnetic interactions in strongly correlated systems. Ann. Phys. 333, 221-271 (2013).

49. Mattis, D. C. The Theory of Magnetism Made Simple (World Scientific, 2006)

50. Mentink, J. H., Balzer, K. \& Eckstein, M. Ultrafast and reversible control of the exchange interaction in Mott insulators. Nat. Commun. 6, 6708 (2015).

51. Tsuji, N., Oka, T., Werner, P. \& Aoki, H. Changing the interaction of lattice fermions dynamically from repulsive to attractive in ac fields. Phys. Rev. Lett. 106, 236401 (2011)

52. Zhao, Z. Y. et al. Ground state and magnetic phase transitions of orthoferrite $\mathrm{DyFeO}_{3}$. Phys. Rev. B 89, 224405 (2014).

53. Marusak, L. A., Messier, R. \& White, W. B. Optical absorption spectrum of hematite $\alpha-\mathrm{Fe}_{2} \mathrm{O}_{3}$, near IR to UV. J. Phys. Chem. Solids 41, 981-984 (1980).

54. Jantzt, W., Sandercock, J. R. \& Wettlingt, W. Determination of magnetic and elastic properties of $\mathrm{FeBO}_{3}$, by light scattering. J. Phys. C: Solid State Phys 9, 2229-2240 (1976).

\section{Acknowledgements}

The research leading to these results was partially funded from the European Commission's 7th Framework Program (FP7/2007-2013) under Grant Agreement 228673 (MAGNONICS) and from EPSRC of the UK under project EP/E055087/1. The work was partially supported by the Netherlands Organization for Scientific Research (NWO), the Foundation for Fundamental Research on Matter (FOM), the European Union's Seventh Framework Program (FP7/2007-2013) grants no. NMP3-LA-2010-246102 (IFOX), no. 280555 (Go-Fast), no. 214810 (FANTOMAS), the European Research Council under the European Union's Seventh Framework Program (FP7/2007-2013)/ERC Grant Agreement No. 257280 (Femtomagnetism) and ERC Grant Agreement 339813(EXCHANGE). The funding support by the Russian Ministry of Education and Science was realized via the Program 'Leading Scientist' Program (Projects 14.B25.31.0025 and 14.750.31.0034). R.V.P. and A.W. acknowledge the support of the joint RFBR-NSFC project (No. 12-02-91172-a, 51211120184, 51511130037) and Bureau of International Cooperation, CAS. A. W. thanks for the support of NSFC project (No. $51372257,51572275)$ and NSFC-NWO joint project (No. 51211130596). A.S. and M.I.K acknowledge support from EU Seventh Framework Program grant agreement No. 281043 (FEMTOSPIN). J.H.M. acknowledges funding from NWO by a Rubicon grant. E.H. is supported by the European Commission (FP7-ICT-2013-613024-GRASP) and EPSRC fellowship (EP/K041215/1)

\section{Author contributions}

R.V.M., V.V.K. and A.V.K. conceived the project. E.H. and R.V.M. designed and built the experimental set up used to study $\mathrm{TmFeO}_{3}$ and $\mathrm{YFeO}_{3}$ samples at the University of Exeter. R.V.M. designed and built the experimental set up at the Radboud University Nijmegen to study the $\mathrm{ErFeO}_{3}, \mathrm{DyFeO}_{3}, \mathrm{FeBO}_{3}$ and $\alpha-\mathrm{Fe}_{2} \mathrm{O}_{3}$ samples. R.V.M. performed all measurements, analysed the data and developed the macroscopic theoretical formalism. A.S. and M.I.K. derived the quantum theory of non-equilibrium exchange. J.M. and M.E. derived the Floquet theory and performed numerical simulations of non-equilibrium exchange. R.V.P. and A.W. prepared the samples. R.V.M. and A.V.K co-wrote the paper with substantial contributions from E.H., V.V.K., R.V.P., A.S., J.M., M.E., M.I.K. and Th.R. The project was coordinated by A.V.K.

\section{Additional information}

Supplementary Information accompanies this paper at http://www.nature.com/ naturecommunications

Competing financial interests: The authors declare no competing financial interests. 
Reprints and permission information is available online at http://npg.nature.com/ reprintsandpermissions/

How to cite this article: Mikhaylovskiy, R. V. et al. Ultrafast optical modification of exchange interactions in iron oxides. Nat. Commun. 6:8190 doi: 10.1038/ncomms9190 (2015). (c) (i) This work is licensed under a Creative Commons Attribution 4.0 International License. The images or other third party material in this article are included in the article's Creative Commons license, unless indicated otherwise in the credit line; if the material is not included under the Creative Commons license, users will need to obtain permission from the license holder to reproduce the material. To view a copy of this license, visit http://creativecommons.org/licenses/by/4.0/ 